\title{
U ma visão geral sobre ontologias: pesquisa sobre definições, tipos, aplicações, métodos de avaliação e de construção
}

\author{
Mauricio B. Almeida \\ M estre em Ciência da Informação \\ Professor assistente da PU C M inas \\ E-mail: mba@pucminas.br
}

\section{Marcello P. Bax}

Doutor em Ciência da Computação

Professor Adjunto da ECI - UFMG

E-mail: bax@ufmg.br

\section{Resumo}

Os estudos sobre a organização da informação têm recebido cada vez mais importância à medida que o número crescente de fontes de dados disponíveis dificulta a recuperação da informação. Nos últimos anos, vários trabalhos têm destacado o uso de ontologias como alternativa para a organização da informação. Este artigo objetiva proporcionar uma visão geral sobre o estado-da-arte no estudo de ontologias. Apresentam-se definições para o termo, uma breve discussão sobre seu significado, tipos de ontologias, propostas para aplicações em diferentes domínios de conhecimento e propostas para a construção de ontologias (metodologias, ferramentas e linguagens).

Palavras-chave

Ontologias; Organização da informação.

An overview about ontologies: survey about definitions, types, applications, evaluation and building methods

\begin{abstract}
Researches on the organization of information have received more and more emphasis as the increased number of available data sources has compromised the retrieval of information. In the past few years, several studies have emphasized the use of ontologies as an alternative to information organization. This paper seeks to provide an overview of the state-of-the-art approaches regarding ontologies. Definitions and a brief discussion about the sense of the term are presented, as well as types of ontologies, proposals of applications in different knowledge domains and suggestions for the building up of ontologies (methodologies, tools and languages).
\end{abstract}

Keywords

Ontologies; Information organization.

Ci. Inf., Brasília, v. 32, n. 3, p. 7-20, set./dez. 2003

\section{IN TRO D U Ç Ã O}

Nos últimos anos, o aumento exponencial dos dados disponíveis tem conferido importância significativa às técnicas de organização da informação. Essas técnicas fazem parte de um corpo de disciplinas que busca melhorias no tratamento de dados, atuando na sua seleção, no seu processamento, na sua recuperação e na sua disseminação.

Diversos tipos de estruturas são utilizados na organização da informação. Estruturas que se organizam a partir da utilização determos são osarquivos deautoridade, glossários edicionários. Estruturas que se organizam com a classificação e a criação de categorias são os cabeçal hos de assunto e os esquemas de classificação (ou taxonomias). A s estruturas quese organizam a partir de conceitos e de seus relacionamentos são as ontologias, ostesaurus eas redes semânticas.

Nos últimos anos, uma abordagem que tem recebido atenção é a utilização de ontologias na organização do conteúdo das fontes de dados. U ma ontologia é criada por especialistas e define as regras que regulam a combinação entre termos e relações em um domínio do conhecimento. O s usuários formulam consultas usando conceitos definidos pela ontologia. 0 que se busca, em última instância, são melhorias nos processos de recuperação da informação.

O ntologias são utilizadas hoje em diversas áreas para organizar a informação (Bateman, 1996; Borgo et alii, 1997; A guado et alii,, 1998; D omingue, 1998; $H$ asman et alii, 1999; Shum; M otta \& Domingue, 2000; Leger et alii, 2000; Kalfoglou, 2001; Vázquez, Valera \& Bellido, 2001; Gandon, 2001; M artin \& Eklund, 2001; A lexaki et alii, 2002). São encontradas na literatura diversas definições para as ontologias, diversos tipos, propostas para aplicação em diferentes áreas de conhecimento e propostas para a construção de ontologias (metodologias, ferramentas elinguagens). Tal diversidade tem dificultado a escolha e a utilização das técnicas disponíveis para a manipulação de ontologias na organização da informação.

0 objetivo desse artigo é sistematizar as principais contribuições, proporcionando uma visão geral do estadoda-arte em ontologias. A pesquisa sobre projetos, 


\section{M auricio B. Almeida / M arcello P. B ax}

metodologias, ferramentas, linguagens e métodos de avaliação apresentada não tem a pretensão de abordar todas as iniciativas existentes. A pesar da preocupação em cobrir os itens mais representativos descritos recentemente na literatura, este estudo não é exaustivo. Por conter muitas abordagens, o trabalho não se aprofunda em nenhuma delas, descrevendo-as sinteticamente.

Este artigo está organizado conforme segue: introdução das ontologias e estudo das diferentes definições, conceitos básicos, características e tipos de ontologias; apresentação de uma pesquisa sobre o uso de ontologias, destacando projetos, repositórios e on tologias conhecidas; abordagem da construção de ontologias, apresentandose metodologias, ferramentas e linguagens, além de métodos para a avaliação dos resultados; finalmente, apresentação das conclusões e indicação de direções para trabalhos futuros.

\section{ONTOLOGIAS: DEFINIÇÕES, CONCEITOS BÁSICOS E TIPOS}

\section{D efinições e conceitos básicos}

$\mathrm{H}$ istoricamente $\mathrm{o}$ termo ontologia tem origem no grego "ontos", ser, e "logos", palavra. 0 termo original éa palavra aristotélica "categoria", que pode ser usada para classificar al guma coisa. A ristóteles apresenta categorias que servem de base para classificar qualquer entidade e introduz ainda o termo "differentia" para propriedades que distinguem diferentes espécies do mesmo gênero. A conhecida técnica de herança é o processo de mesclar differentias definindo categorias por gênero.

Em seu sentido filosófico, trata-se de um termo relativamente novo, introduzido com o objetivo de distinguir o estudo do ser como tal. 0 Dicionário 0 xford deFilosofia defineontologia como "[...] 0 termo derivado da palavra grega que significa 'ser', mas usado desde o século XVII para denominar 0 ramo da metafísica que diz respeito àquilo que existe" (Blackburn \& M arcondes, 1997).

0 termo ontologia tem um sentido especial em organização da informação, diferente daquele tradicional adotado na filosofia. São diversas as definições apresentadas na literatura e existem contradições.

De forma simples, para elaborar ontologias, definem-se categorias para as coisas que existem em um mesmo domínio. Ontologia é um "catálogo de tipos de coisas" em que se supõe existir um domínio, na perspectiva de uma pessoa que usa uma determinada linguagem (Sowa, 1999). Trata-se de "uma teoria que diz respeito a tipos de entidades e, especificamente, a tipos de entidades abstratas que são aceitas em um sistema com uma linguagem" (M erriam-W ebster; G ove, 2002* apud C orazzon, 2002, p. 1).

U ma das definições mais conhecidas para ontologias é apresentada por G ruber (1996),** apud Corazzon, 2002, p. 1:

"U ma ontologia é uma especificação explícita de uma conceitualização. [...] Em tal ontologia, definições associam nomes de entidades no universo do discurso (por exemplo, classes, relações, funções etc. com textos que descrevem 0 que os nomes significam e os axiomas formais que restringem a interpretação e o uso desses termos) [...]."

0 termo conceitualização corresponde a uma coleção de objetos, conceitos e outras entidades que se assume existirem em um domínio e os relacionamentos entre eles (G enesereth \& N ilsson, 1987). U ma conceitualização é uma visão abstrata e simplificada do mundo que se deseja representar.

A definição proposta por $\mathrm{G}$ ruber é discutida em Guarino \& G iaretta (1995)***, apud Corazzon, 2002, p. 1:

“[...] um ponto inicial nesse esforço de tornar claro 0 termo será uma análise da interpretação adotada por Gruber. 0 principal problema com tal interpretação é que ela é baseada na noção conceitualização, a qual não corresponde à nossa intuição. [...] U ma conceitualização é um grupo de relações extensionais descrevendo um 'estado das coisas' particular, en quanto a noção que temos em mente é uma relação intensional, nomeando algo como uma rede conceitual a qual se superpõe a vários possíveis 'estados das coisas'."

U ma definição intensional consiste de uma lista de características do conceito. Por exemplo, lâmpada incandescente é a lâmpada elétrica que emite luz a partir do aquecimento de um filamento pela corrente elétrica. A lâmpada incandescente é definida como o auxílio do gênero mais próximo (lâmpada elétrica) e de suas características. U ma definição extensional é uma en umeração de aspectos de todas as espécies que são do mesmo nível de abstração. Por exemplo, os planetas do sistema solar são M ercúrio, Vênus, Terra, M arte, Júpiter, Saturno, U rano, N etuno e Plutão (I SO-Standard 704).

\footnotetext{
* MerRiam-Webster; GOVE, P. B. Webster's Third New International Dictionary. U nabridged. $\mathrm{N}$ ew York: M erriam-W ebster, 2002. $2.783 \mathrm{p}$.

** GRU BER, T. (1996). W hat is an Ontology? Disponível em: «ttp:/ Lwww-ksl.stanford.edu/kst/ what-is-an-ontology.html>. A cesso em: 14 set. 2002.

*** GUARIN O, N.; GIARETTA, P. (1995). O ntologies and KBs, towards a terminological clarification. Disponível em: $<$ http:// www.ladseb.pd.cnr.it/infor/O ntology/Papers/KBKS95.pdf>. A cesso em: 13 jul. 2002.
} 
Guarino (1998) revê a definição de conceitualização fazendo uso do aspecto intensional, para obter uma interpretação mais satisfatória:

"[...] ontologia se refere a um artefato constituído por um vocabulário usado para descrever uma certa realidade, mais um conjunto de fatos explícitos e aceitos que dizem respeito ao sentido pretendido para as palavras do vocabulário. Este conjunto de fatos tem a forma da teoria da lógica de primeira ordem, onde as palavras do vocabulário aparecem como predicados unários ou binários."

0 vocabulário formado por predicados lógicos forma a rede conceitual que confere 0 caráter intensional às ontologias. A ontologia define as regras que regulam a combinação entre os termos e as relações. As relações entre os termos são criadas por especialistas, e os usuários formulam consultas usando os conceitos especificados. U ma ontologia define assim uma "linguagem" (conjunto de termos) que será utilizada para formular consultas.

B orst $(1997$, p. 12) apresenta uma definição simples e completa, que será adotada neste trabal ho: "U ma ontologia é uma especificação formal e explícita de uma conceitualização compartilhada". N essa definição, "formal" significa legível para computadores; "especificação explícita" diz respeito a conceitos, propriedades, relações, funções, restrições, axiomas, explicitamente definidos; "compartilhado" quer dizer conhecimento consensual; e "conceitualização" diz respeito a um modelo abstrato de algum fenômeno do mundo real.

Discussões podem ser encontradas em Guarino \& G iaretta (1995), que apresentam diferentes sentidos para o termo em relação a níveis de abstração. O utras definições para o termo são encontradas em Albertazi (1996), N eches et alii (1991), W acheet alii (2001), U schold \& Gruninger (1996) e Chandrasekaran, Johnson \& Benjamins (1999). Para uma discussão detalhada, considerações e críticas, ver Guarino (1996) e Guarino (1998). O zkural (2001) referese a ontologias como uma teoria de classificação.

M esmo sem um consenso sobre sua definição, as ontologias apresentam características comuns. A seção seguinte apresenta as principais características das ontologias e uma breve revisão de literatura sobre como podem ser classificadas.

\section{Características e tipos de ontologias}

As ontologias não apresentam sempre a mesma estrutura, mas existem características e componentes básicos comuns presentes em grande parte delas. M esmo apresentando propriedades distintas, é possível identificar tipos bem definidos.
O s componentes básicos de uma ontologia são classes (organizadas em uma taxonomia), relações (representam o tipo de interação entre os conceitos de um domínio), axiomas (usados para modelar sentenças sempre verdadeiras) e instâncias (utilizadas para representar elementos específicos, ou seja, os próprios dados) (G ruber, 1996; N oy \& Guinness, 2001).

Algumas das propostas definem tipos de ontologias relacionando-as à sua função (M izoguchi, V anwelkenuysen \& Ikeda, 1995), ao grau de formalismo de seu vocabulário (U schold \& G runinger, 1996), à sua aplicação (Jasper \& U schold, 1999) e à estrutura e conteúdo daconceitualização (V an H eijist, Schreiber \& W ielinga, 1997), (H aav \& Lubi, 2001). A tabela 1, a seguir, sintetiza cada abordagem.

M esmo sem um consenso, observa-se que os tipos apresentados guardam semelhanças entre suas funções. Conhecidos os principais tipos e características, podese buscar ontologias existentes adequadas à utilização desejada. A seção seguinte apresenta uma pesquisa sobre a utilização de ontologias em diversos projetos citados na literatura, além de repositórios e ontologias conhecidas.

\section{PESQUISA SOBRE A UTILIZAÇÃO DE ONTOLOGIAS}

\section{Projetos que fazem uso das ontologias}

O ntologias são utilizadas em projetos de domínios como gestão do conhecimento, comércio eletrônico, processamento de linguagens naturais, recuperação da informação na $W$ eb, de cunho educacional, entre outros. As tabelas 2, 3, 4, 5 e 6, a seguir, apresentam exemplos de projetos que utilizam ontologias e uma descrição sintética.

\section{Exemplos de ontologias e repositórios de ontologias}

Existem ontologias disponíveis para uso ou para modelar a construção de outras ontologias. $N$ a seqüência, as tabelas 7, 8, 9 e 10, a seguir, apresentam exemplos dessas ontologias e uma descrição sintética de cada uma delas. A tabela 11, a seguir, apresenta alguns repositórios de ontologias disponíveis na Internet.

\section{CONSTRUINDO ONTOLOGIAS: METODOLOGIAS, FERR AMENTAS, LINGUAGENS E AVALIAÇÃO}

\section{$M$ etodologias}

M etodologias têm sido desenvolvidas no intuito de sistematizar a construção e a manipulação de ontologias (López, 1999). Existem metodologias para a construção de ontologias, para construção de ontologias em grupo, 
TABELA 1

Tipos de ontologias

\begin{tabular}{|c|c|c|}
\hline Abordagem & Classificaçăo & Descrição \\
\hline \multirow{3}{*}{$\begin{array}{l}\text { Quanto à função } \\
\text { Mizoguchi, } \\
\text { Vanwelkenhuysen \& } \\
\text { Ikeda (1995) }\end{array}$} & Ontologias de domínio & $\begin{array}{l}\text { Reutilizáveis no domínio, fornecem vocabulário sobre conceitos, seus relacionamentos, } \\
\text { sobre atividades e regras que os governam. }\end{array}$ \\
\hline & Ontologias de tarefa & $\begin{array}{l}\text { Fornecem um vocabulário sistematizado de termos, especificando tarefas que podem ou não } \\
\text { estar no mesmo domínio. }\end{array}$ \\
\hline & Ontologias gerais & $\begin{array}{l}\text { Incluem um vocabulário relacionado a coisas, eventos, tempo, espaço, casualidade, } \\
\text { comportamento, funçóes etc. }\end{array}$ \\
\hline \multirow{4}{*}{$\begin{array}{l}\text { Quanto ao grau de } \\
\text { formalismo } \\
\text { Uschold \& Gruninger } \\
\text { (1996) }\end{array}$} & Ontologias altamente informais & Expressa livremente em linguagem natural. \\
\hline & Ontologias semi-informais & Expressa em linguagem natural de forma restrita e estruturada. \\
\hline & Ontologias semiformais & Expressa em uma linguagem artificial definida formalmente. \\
\hline & Ontologia rigorosamente formal & Os termos säo definidos com semântica formal, teoremas e provas. \\
\hline \multirow{3}{*}{$\begin{array}{l}\text { Quanto à aplicação } \\
\text { Jasper \& Uschold } \\
\text { (1999) }\end{array}$} & Ontologias de autoria neutra & $\begin{array}{l}\text { Um aplicativo é escrito em uma única língua e depois convertido para uso em diversos } \\
\text { sistemas, reutilizando-se as informaçoes. }\end{array}$ \\
\hline & Ontologias como especificação & $\begin{array}{l}\text { Cria-se uma ontologia para um domínio, a qual é usada para documentação e manutenção no } \\
\text { desenvolvimento de softwares. }\end{array}$ \\
\hline & $\begin{array}{l}\text { Ontologias de acesso comum à } \\
\text { informação }\end{array}$ & $\begin{array}{l}\text { Quando o vocabulário é inacessível, a ontologia torna a informaçấo inteligível, } \\
\text { proporcionando conhecimento compartilhado dos termos. }\end{array}$ \\
\hline \multirow[t]{3}{*}{$\begin{array}{l}\text { Quanto à estrutura } \\
\text { Haav \& Lubi (2001) }\end{array}$} & Ontologias de alto nível & $\begin{array}{l}\text { Descrevem conceitos gerais relacionados a todos os elementos da ontologia (espaço, tempo, } \\
\text { matéria, objeto, evento, ação etc.) os quais são independentes do problema ou domínio. }\end{array}$ \\
\hline & Ontologias de domínio & $\begin{array}{l}\text { Descrevem o vocabulário relacionado a um domínio, como, por exemplo, medicina ou } \\
\text { automóveis. }\end{array}$ \\
\hline & Ontologias de tarefa & $\begin{array}{l}\text { Descrevem uma tarefa ou atividade, como, por exemplo, diagnósticos ou compras, mediante } \\
\text { inserção de termos especializados na ontologia. }\end{array}$ \\
\hline \multirow{7}{*}{$\begin{array}{l}\text { Quanto ao conteúdo } \\
\text { Van-Heijist, } \\
\text { Schreiber \& Wielinga } \\
(2002)\end{array}$} & Ontologias terminológicas & $\begin{array}{l}\text { Especificam termos que serão usados para representar o conhecimento em um domínio (por } \\
\text { exemplo, os léxicos). }\end{array}$ \\
\hline & Ontologias de informação & $\begin{array}{l}\text { Especificam a estrutura de registros de bancos de dados (por exemplo, os esquemas de } \\
\text { bancos de dados). }\end{array}$ \\
\hline & $\begin{array}{l}\text { Ontologias de modelagem do } \\
\text { conhecimento }\end{array}$ & $\begin{array}{l}\text { Especificam conceitualizaçôes do conhecimento, têm uma estrutura interna semanticamente } \\
\text { rica e são refinadas para uso no domínio do conhecimento que descrevem. }\end{array}$ \\
\hline & Ontologias de aplicação & Contêm as definiçōes necessárias para modelar o conhecimento em uma aplicação. \\
\hline & Ontologias de dornínio & $\begin{array}{l}\text { Expressamn conceitualizaçớes que são específicas para um determinado domínio do } \\
\text { conhecimento. }\end{array}$ \\
\hline & Ontologias genéricas & $\begin{array}{l}\text { Similares às ontologias de domínio, mas os conceitos que as definem são considerados } \\
\text { genéricos e comuns a vários campos. }\end{array}$ \\
\hline & Ontologias de representação & $\begin{array}{l}\text { Explicam as conceitualizaçốes que estão por trás dos formalismos de representação do } \\
\text { conhecimento. }\end{array}$ \\
\hline
\end{tabular}

TABELA 2

Projetos relacionados à gestão do conhecimento

\begin{tabular}{|c|c|}
\hline Projetos & Breve descriçăo \\
\hline $\begin{array}{l}\text { CoMMA(Corporate } \\
\text { Memory Management } \\
\text { through Agents) }\end{array}$ & $\begin{array}{l}\text { A memória corporativa é descrita como "Web semântica corporativa," e o resultado é uma ontologia; utiliza agentes } \\
\text { inteligentes para construir uma arquitetura de informação distribuída e agentes locais por meio dos quais os usuários acessam } \\
\text { os recursos disponíveis (Gandon, 2001). }\end{array}$ \\
\hline $\begin{array}{l}\text { Marchmont Observatory } \\
\text { Semantic Search Service }\end{array}$ & $\begin{array}{l}\text { Projeto relacionado à educaçấo continuada, consiste de um portal onde sáo construídas ontologias associadas à educaçấo. } \\
\text { Estas ontologias indexam um banco de dados de "melhores práticas" com sumários sobre educação (Domingue , 1998). }\end{array}$ \\
\hline $\begin{array}{l}\text { MGT (Medical } \\
\text { Guideline Technology) }\end{array}$ & $\begin{array}{l}\text { Adequada para uso na Web, a ferramenta constrói uma hierarquia de ontologias médicas e de suporte, as quais podem ser } \\
\text { integradas ao banco de dados de um paciente (Hasman et alii, 1999). }\end{array}$ \\
\hline MyPlanet & $\begin{array}{l}\text { Serviço personalizado para a Web, onde o usuário submete um e-mail sobre seus interesses de pesquisa; este e-mail é } \\
\text { adaptado a estruturas ontológicas, e uma página da Web é produzida; os usuários são notificados sobre o assuntos de seu } \\
\text { interesse (Kalfoglon, 2001). }\end{array}$ \\
\hline PotMon & $\begin{array}{l}\text { Projeto da área de saíde que trata de questóes médicas e administrativas no gerenciamento de pacientes; possui um fórum } \\
\text { para discussões e um motor de consultas baseado em ontologias; possibilita recuperar conhecimento sobre determinado } \\
\text { assunto médico (Motta, Buckingham-Shum \& Domingue, 2000). }\end{array}$ \\
\hline PlanetOnto & $\begin{array}{l}\text { Possui um servidor de noticias para a Web que facilita a comunicaçäo em empresas; à medida que o arquivo de noticias no } \\
\text { servidor torna-se maior, ocorrem problemas de gestão (semântica, recuperação de informação, personalização etc.). Um } \\
\text { conjunto de ferramentas chamado PlanetOnto permite representar formalmente os documentos utilizando-se ontologias } \\
\text { (Domingue \& Motta, 1999). }\end{array}$ \\
\hline
\end{tabular}


TABELA 3

Projetos relacionados a comércio eletrônico

\begin{tabular}{|c|c|}
\hline Projeto & Breve descriçăo \\
\hline $\begin{array}{l}\text { MKBEEM (Multilingual } \\
\text { Knowledge Based European } \\
\text { Electronic Marketplace) }\end{array}$ & $\begin{array}{l}\text { Proporciona habilidades multilingüísticas ao fluxo de informação em portais B2C (Business-to-consumer): permite } \\
\text { manutenção semi-automática de catálogos de produtos, tradução automática e interpretação da linguagem natural nas } \\
\text { requisiçôes de usuários; a interatividade é obtida com o uso de serviços de navegaçâo e entradas em linguagem } \\
\text { natural (Leger et alii, 2000). }\end{array}$ \\
\hline $\begin{array}{l}\text { SMART-EC (Smart-EC Support } \\
\text { for Mediation And brokering for } \\
\text { Electronic Commerce) } \\
\end{array}$ & $\begin{array}{l}\text { Plataforma de intermediaçấo baseada em ontologias que fomece serviços para a Intemet, como troca de informaçốes } \\
\text { entre provedores e usuánios finais, definição e implementação de ciclo de vida de serviços e a possibilidade de } \\
\text { compras em diversos sites a partir de interface única (Vázquez, Valera \& Bellido, 2001). }\end{array}$ \\
\hline
\end{tabular}

TABELA 4

\section{Projetos relacionados ao processamento de linguagens naturais}

\begin{tabular}{|c|c|}
\hline Projeto & Breve descriçăo \\
\hline Oncoterm & $\begin{array}{l}\text { Facilita a tradução de textos médicos sobre oncologia mediante uma ontologia baseada em textos especializados e dicionários } \\
\text { médicos; os conceitos são organizados em categorias e representados por esquemas (Moreno \& Hernández, 2000). }\end{array}$ \\
\hline Gazelle & $\begin{array}{l}\text { Traduz textos japoneses, árabes e espanhóis para o inglês; inclui processamento e análise semântica das línguas, geração de } \\
\text { sentenças em inglês, construção de ontologias interlíngua e criaçăo de léxicos para japonês, árabe, espanhol e inglês (Germann, } \\
\text { 1998). }\end{array}$ \\
\hline Mikrokosmos & $\begin{array}{l}\text { Mecanismo em que textos em linguagem natural são traduzidos para textos no formato interlíngua (linguagem neutra, chamada } \\
\text { TMR-text meaning representation); a ligação da ontologia com o TMR é feita por meio de um léxico, no qual os significados dos } \\
\text { itens são definidos por conceitos ontológicos (Beale, Nirenburg \& Mahesh, 1995). }\end{array}$ \\
\hline PANGLOSS & $\begin{array}{l}\text { Possui um sistema baseado em exemplos e um sistema de transferência léxica que trabalham em paralelo propondo traduçôes das } \\
\text { entradas; o resultado ou tradução final é selecionada por um modelo estatístico (Frederking, 1994) }\end{array}$ \\
\hline $\begin{array}{l}\text { KPML (Komet- } \\
\text { Penman } \\
\text { MultiLingual) }\end{array}$ & $\begin{array}{l}\text { Desenvolve gramáticas e gera linguagens naturais; oferece um ambiente de desenvolvimento gráfico para a construção, } \\
\text { manutençăo e uso de gramáticas para diversas línguas (inglês, alemão, holandês, chinês, espanhol, russo, búlgaro e checo) } \\
\text { (Bateman, 1996). }\end{array}$ \\
\hline Ontogeneration & $\begin{array}{l}\text { Gera textos em espanhol no domínio da química e utiliza linguagens naturais para responder a consultas sobre grupos, elementos e } \\
\text { propriedades químicas; utiliza uma ontologia da química (Chemicals), uma ontologia lingüística e uma gramática em espanhol } \\
\text { (Aguado et alii, 1998). }\end{array}$ \\
\hline Penman & $\begin{array}{l}\text { Gera sentenças em linguagem natural a partir de uma entrada não lingüística, aceita várias notações na entrada e foi projetado para } \\
\text { o uso por pessoas com vários graus de sofisticaçăo lingüística e computacional (Teich \& Bateman, 1993). }\end{array}$ \\
\hline TechDoc & $\begin{array}{l}\text { Gera documentos técnicos multilinguí́sticos (inglês, alemão e francês) a partir de uma representação independente construída pela } \\
\text { análise comparativa de partes dos manuais técnicos e linguísticos disponíveis em diferentes línguas (Rősner \& Stede, 1994). }\end{array}$ \\
\hline
\end{tabular}

TABELA 5

\section{Projetos relacionados à recuperação da informação na $W$ eb}

\begin{tabular}{|l|l|}
\hline \multicolumn{1}{|c|}{ Projeto } & \multicolumn{1}{c|}{ Breve descriçäo } \\
\hline OntoSeek & $\begin{array}{l}\text { Recupera informaçōes de catálogos de produtos on-line utilizando um sistema de agentes inteligentes, um mecanismo de } \\
\text { casamento de padrăo baseado em ontologias para tratar o conteúdo e um formalismo para representaçăo (Borgo et alii, 1997). }\end{array}$ \\
\hline WebKB-2 & $\begin{array}{l}\text { Permite que usuários da Web recuperem e adicionem conhecimento em uma base compartilhada; permite a publicação de } \\
\text { informaçốes automaticamente recuperáveis e comparáveis com as de outros usuários (Martin \& Eklund, 2001). }\end{array}$ \\
\hline $\begin{array}{l}\text { C-Web- } \\
\text { Community Web }\end{array}$ & $\begin{array}{l}\text { Formaliza o conhecimento comum utilizado por comunidades da Web; a limitação é conseguir um ponto de acesso único para as } \\
\text { várias fontes de informação das comunidades (Alexaki et alii, 2002). }\end{array}$ \\
\hline $\begin{array}{l}\text { SEAL (Semantic } \\
\text { Portal) }\end{array}$ & $\begin{array}{l}\text { Possibilita o descnvolvimento de portais semânticos a partir de abordagem bascada cm ontologias; cxplora o aspecto semântico por } \\
\text { meio do fornecimento e acesso a informaçóes em um portal (Maedche et alii, 2001) }\end{array}$ \\
\hline
\end{tabular}

TABELA 6

Projetos relacionados à educação

\begin{tabular}{|l|l|}
\hline \multicolumn{1}{|c|}{ Projeto } & \multicolumn{1}{c|}{ Breve descrição } \\
\hline RichODL & $\begin{array}{l}\text { Ambiente de aprendizado na Web desenvolvido para treinar estudantes na modelagem e simulação de ambientes dinâmicos; } \\
\text { ontologias săo usadas para descrever o domínio físico dos sistemas modelados, além de suas correlaços (Zdrahal et alii, 2000). }\end{array}$ \\
\hline Smartrainer & $\begin{array}{l}\text { Sistema de treinamento automático no domínio do fornecimento de energia; treina funcionários de empresas de energia para a } \\
\text { recuperação de acidentes em subestaçốes elétricas (Jin } \text { et alii, 1997). }\end{array}$ \\
\hline $\begin{array}{l}\text { SchoolOnto } \\
\text { Scholarly } \\
\text { Ontologies Project }\end{array}$ & $\begin{array}{l}\text { Biblioteca digital baseada em ontologias que possibilita interpretar domínios; auxilia na modelagem de pesquisas dinâmicas que } \\
\text { carecem de ferramentas para tratar inconsistências; permite discutir a contribuição do documento para a literatura da área por meio } \\
\text { de uma rede semântica (Shum, Motta \& Domingue, 2000). }\end{array}$ \\
\hline
\end{tabular}


TABELA 7

O ntologias de alto nível

\begin{tabular}{|c|c|}
\hline Ontologia & Breve descriçäo \\
\hline SOWA & $\begin{array}{l}\text { Inclui categorias básicas derivadas de várias fontes (lógica, linguística, filosofia, inteligência artificial), O conceito de mais alto nível é o } \\
\text { tipo "universal", e o de mais baixo nível é o tipo "absurdo". Os subtipos do tipo universal são conceitos primitivos que combinados } \\
\text { geram novos conceitos (Sowa, 1999). }\end{array}$ \\
\hline Guarino & $\begin{array}{l}\text { As categorias são divididas em "universais" e "particulares". Os particulares são conceitos abstratos, por exemplo, um objeto ou um } \\
\text { evento, que podem ser instanciados em elementos específicos. Os universais săo também conceitos abstratos (por exemplo, tịo e papel) } \\
\text { que podem ser instanciados em particulares. É possível provar que cada propriedade pode ser instanciada em pelo menos um dos níveis } \\
\text { da hierarquia (Guarino \& Welty, 2000). }\end{array}$ \\
\hline $\begin{array}{l}\text { IEEE } \\
\text { Standard }\end{array}$ & $\begin{array}{l}\text { É um padrăo da IEEE chamado Standard Upper Merged Ontology, que tem o propósito de especificar uma ontologia de alto nível para } \\
\text { utilizaçẫo em aplicaçốes de interoperabilidade, busca, recuperação, inferências automáticas, processamento de linguagens naturais etc. }{ }^{1}\end{array}$ \\
\hline
\end{tabular}

${ }^{1}$ Disponível na Internet em http:// reliant.teknowledge.com/DAM L/

SU 0.daml e http:// suo.ieee.org/

TABELA 8

\section{O ntologias lingüísticas}

\begin{tabular}{|c|c|}
\hline Ontologia & Breve descrição \\
\hline CoreLex & $\begin{array}{l}\text { É uma ontologia léxico-semântica que contém } 126 \text { tipos semânticos, cobre cerca de } 40 \text { mil substantivos e define um grande } \\
\text { número de classes polissêmicas, derivadas de análise do Wordnet (Buitelaar, 2001). }\end{array}$ \\
\hline $\begin{array}{l}\text { EDR Electronic } \\
\text { Dictionary }\end{array}$ & $\begin{array}{l}\text { Desenvolvido para processamento de linguagem natural, integra relações entre entradas léxicas e seus conceitos na forma de uma } \\
\text { hierarquia e de relações semânticas, agrupados em um banco de dados de onde os conceitos são extraídos. É composto por cinco } \\
\text { grandes tipos de dicionários (Ogino et alii, 1997). }\end{array}$ \\
\hline EuroWordNet & $\begin{array}{l}\text { Trata-se de um conjunto de dicionários para línguas européias (holandês, italiano, espanhol, alemão, francês, tcheco, estoniano), } \\
\text { organizados a partir de grupos de sinônimos e das relações entre eles. Cada dicionário é conectado a um índice interlíngua que } \\
\text { acessa uma ontologia semântica de alto nível, possibilitando navegar de uma palavra em uma língua para a mesma palavra em } \\
\text { outra língua. Pode ser utilizado para recuperação da informação multilíngüe (Vossen, 1998). }\end{array}$ \\
\hline $\begin{array}{l}\text { GT (Goi-Taikei's) } \\
\text { ontology }\end{array}$ & $\begin{array}{l}\text { Trata-se de um léxico de } 40 \text { mil palavras japonesas que consiste de uma ontologia, um dicionário semântico de palavras e um } \\
\text { dicionário semântico da estrutura. A ontologia classifica conceitos expressando as relações entre as palavras. O significado das } \\
\text { palavras é dado por uma hierarquia semântica, em que os nós representam uma classe semântica e as arestas representam } \\
\text { relações é-um ou tem-um (Bond et alii, 2001). }\end{array}$ \\
\hline $\begin{array}{l}\text { GUM(Generalized } \\
\text { Upper Model })\end{array}$ & $\begin{array}{l}\text { Ontologia para processamento de linguagens naturais que simplifica a interface entre conhecimento de um domínio e recursos } \\
\text { linguísticos genéricos. Consiste de duas hierarquias: uma contém todos os conceitos, e a outra contém todos os papéis (Bateman, } \\
\text { Magnini \& Fabris, 1995). }\end{array}$ \\
\hline Wordnet & $\begin{array}{l}\text { Trata-se de um grande banco de dados léxico para a língua inglesa, em que são organizados } 70 \text { mil grupos de sinônimos, cada } \\
\text { um representando um conceito léxico. Os sinônimos são conectados por relações, e o léxico é dividido em substantivos, verbos, } \\
\text { adjetivos e advérbios (Miller, 1995). }\end{array}$ \\
\hline
\end{tabular}

TABELA 9

O ntologias para empresas

\begin{tabular}{|l|l|}
\hline \multicolumn{1}{|c|}{ Ontologia } & \multicolumn{1}{c|}{ Breve descriçẫo } \\
\hline Enterprise Ontology & $\begin{array}{l}\text { Trata-se de uma coleção de termos e definições relevantes para empresas e negócios, incluindo conhecimento sobre atividades, } \\
\text { processos, organizaçöes, estratégias e marketing (Uschold et alii, 1998). }\end{array}$ \\
\hline $\begin{array}{l}\text { TOVE (Toronto } \\
\text { Virtual Enterprise) }\end{array}$ & $\begin{array}{l}\text { Tem por objetivo criar um modelo de dados que forneça uma terminologia compartilhada para as empresas, definir o significado } \\
\text { de cada termo, implementar semântica em um grupo de axiomas que permitem deduçöes sobre questöes de senso comum na } \\
\text { empresa e definir uma simbologia para representação de um termo ou conceito em um contexto gráfico (Fox,1981). }\end{array}$ \\
\hline
\end{tabular}

TABELA 10

O ntologias para domínios específicos

\begin{tabular}{|l|l|}
\hline \multicolumn{1}{|c|}{ Ontologia } & \multicolumn{1}{c|}{ Breve descriçäo } \\
\hline GALEN & $\begin{array}{l}\text { Representado em linguagem formal, é baseado em um modelo semântico para terminologia clínica CORE (Coding Reference } \\
\text { Model); reúne conceitos clínicos elementares, relações e controla como podem ser feitas as combinações para a formação de } \\
\text { conceitos complexos (Rector et alii, 1995). }\end{array}$ \\
\hline $\begin{array}{l}\text { UMLS (The Unified } \\
\text { System) }\end{array}$ & $\begin{array}{l}\text { Conecta vocabulário biomédico de fontes diversas (terninologia clínica, fontes sobre drogas etc.) em diversas línguas. } \\
\text { Consiste de um metatesaurus com informação semântica sobre os conceitos, seus nomes e relaçốes; de uma rede semântica de } \\
\text { categorias genéricas às quais os termos do metatesaurus säo atribuídos; e um léxico especializado que contém informação } \\
\text { sintática sobre termos biomédicos (Pisanelli, Gangemi \& Steve, 1998). }\end{array}$ \\
\hline CHEMICALS & $\begin{array}{l}\text { Ontologia do domínio da química que contém conhecimento sobre elementos químicos e estruturas cristalinas (Fernández- } \\
\text { Lopez et alii, 1999) }\end{array}$ \\
\hline
\end{tabular}


TABELA 11

\section{Repositórios de ontologias}

\begin{tabular}{|l|l|}
\hline \multicolumn{1}{|c|}{ Repositório } & \multicolumn{1}{c|}{ Breve descrição } \\
\hline DAML & $\begin{array}{l}\text { Repositónio com 192 ontologias, implementadas em DAML+OI, classificadas de acordo com diferentes critérios (URI, } \\
\text { data de submissão, palavras-chave etc.) }\end{array}$ \\
\hline Ontolingua Server & $\begin{array}{l}\text { Armazena aproximadamente 50 ontologias escritas em Ontolingua, além de informações e estatísticas (número de classes, } \\
\text { relações e axiomas) }\end{array}$ \\
\hline Universal Repository: & $\begin{array}{l}\text { Armazena cerca de } 50 \text { ontologias para educadores e escolas, classificadas por áreas de conhecimento (história, } \\
\text { computação, literatura etc.) }\end{array}$ \\
\hline
\end{tabular}

para aprendizado sobre a estrutura de ontologias e para a integração de ontologias. Baseado nos estudos de Corcho, Fernán dez-López \& Gomez-Pérez (2001), apresentam-se várias metodologias para construção de ontologias. As tabelas 12, 13, 14 e 15, a seguir, sintetizam os comentários sobre as metodologias, proporcionando uma visão geral de seu funcionamento.

As metodologias apresentadas possuem abordagens e características diversas. N ão parece provável a unificação das propostas em uma única metodologia. Para verificar a utilidade das metodologias e compará-las, é necessário avaliar a ontologia resultante da aplicação de cada metodologia. A lém de metodologias, existem ferramentas utilizadas para a construção de uma ontologia.

\section{Ferramentas para a construção de ontologias}

Por se tratar de uma tarefa dispendiosa, qualquer apoio na construção de ontologias pode representar ganhos significativos. Exemplos de ferramentas para a construção de ontologias são apresentados a seguir, na tabela 16.

Critérios devem ser definidos para que as ferramentas de construção de ontologias possam ser comparáveis. Em geral, as ferramentas utilizam linguagens de representação para a construção das ontologias, as quais são apresentadas na seção seguinte.

\section{Linguagens para a construção de ontologias}

Alguns exemplos de linguagens que se prestam à construção de ontologias são apresentados a seguir, na tabela 17.

Critérios devem ser definidos para que se possa comparar as linguagens para construção de ontologias apresentadas nessa seção. W ache et alii (2001) apresentam um comparativo entre as linguagens sobre diversos aspectos (operadores, axiomas, declarações etc.). A seção seguinte apresenta uma breve pesquisa sobre métodos para a avaliação de ontologias.

\footnotetext{
${ }^{2}$ Disponível na Internet em http://www.daml.org/ontologies/

3 Disponível na Internet em www-ksl-svc.stanford.edu:5915/

${ }^{4}$ Disponível na Internet em http://www.ist-universal.org/
}

\section{M étodos de avaliação para ontologias}

Propostas para a avaliação de ontologias são encontradas na literatura, mas parecem existir poucas metodologias formais. A construção de ontologias é ainda mais artesanal do que científica (Jones et alii, 1998), e não existem propostas unificadas, sendo que grupos diferentes utilizam diferentes abordagens (Fernandez, 1999). Essa diversidade pode ser um fator que dificulta a formulação de metodologias de avaliação formais.

Algumas questões básicas para a avaliação de ontologias são: Q uais são os mecanismos para interagir com as ontologias? Q ual é o formalismo de representação do conhecimento utilizado? A ontologia é bem documentada? A ontologia foi avaliada sobre o ponto de vista técnico?

Gómez-Perez (1999) apresenta critérios que podem ser utilizados para avaliar ontologias. Os passos apresentados para a avaliação focalizam-se sobre os conceitos e definições que compõem a ontologia:

- Verificar a estrutura ou arquitetura da ontologia: as definições são construídas seguindo os critérios de projeto?

- Verificar a sintaxe das definições: existem estruturas ou palavras-chave sintaticamente incorretas nas definições?

- Verificar o conteúdo das definições: o que a ontologia define ou não? 0 que define incorretamente? 0 que pode ser inferido e o que não pode?

A tabela 18 apresenta, a seguir, exemplos de mecanismos formais para a avaliação de ontologias. 
TABELA 12

Metodologias para construção de ontologias

\begin{tabular}{|c|c|}
\hline Meto & Breve descrição \\
\hline Cyc & $\begin{array}{l}\text { iento implícito e explícito das diferentes fontes, e, quando já se tem conhecimento suficiente na } \\
\text { ser obtido por ferramentas que utilizam linguagem natural (Lenat \& Guha, 1990). }\end{array}$ \\
\hline $\begin{array}{l}\text { USCHOLDe } \\
\text { KING }\end{array}$ & $\begin{array}{l}\text { cósito, os conceitos e relacionamentos entre os conceitos, além dos termos utilizados para codificar a ontologia e, em } \\
\text { entá-la (Uschold \& King, 1996). }\end{array}$ \\
\hline $\begin{array}{l}\text { GRÜNINGER } \\
\text { e FOX }\end{array}$ & $\begin{array}{l}\text { stodo formal que identifica cenários para uso da ontologia, utiliza questð̃es em linguagem natural para determinação do escopo da } \\
\text { tologia, executa a extração sobre os principais conceitos, propriedades, relaçōes e axiomas, definidos em PROLOG (Grüninger \& } \\
\text { X, 1995). }\end{array}$ \\
\hline KACTUS & $\begin{array}{l}\text { étodo recursivo que consiste em uma proposta inicial para uma base de conhecimento; quando é necessária uma nova base em } \\
\text { mínio similar, generaliza-se a primeira base em uma ontologia adaptada a ambas aplicaçōes; quanto mais aplicaçōes, mais genérica } \\
\text { ntologia (Bernaras, Laresgoiti \& Corera, 1996). }\end{array}$ \\
\hline Methe & $\begin{array}{l}\text { genharia sobre outra utilizando-se o conhecimento do domínio; as atividades principais são } \\
\text { malizaçấ, implementaçăo e manutençâo (Fernández-Lopez et alii, 1999). }\end{array}$ \\
\hline Sensus & $\begin{array}{l}\text { Constrói ontologias a partir de outras ontologias, identificando os termos relevantes para o domínio e ligando-os à ontologia mais } \\
\text { abrangente (Sensus, com } 50 \text { mil conceitos); um algoritmo monta a estrutura hierárquica do domínio (Swartout et alii, 1996) }\end{array}$ \\
\hline On-to- & $\begin{array}{l}\text { Auxilia a administração de conceitos em organizaçōes, identificando metas para as ferramentas de gestão do conhecimento e } \\
\text { utilizando cenários e contribuiçôes dos provedores / clientes de informação da organização (Staab et alii, 2001). }\end{array}$ \\
\hline
\end{tabular}

TABELA 13

Metodologias para construção de ontologias em grupo

\begin{tabular}{|l|l|}
\hline Metodologia & \multicolumn{1}{c|}{ Breve descriçẩo } \\
\hline CO4 & $\begin{array}{l}\text { Permite discussão sobre conhecimento introduzido em bases de conhecimento compartilhadas, que correspondem a ontologias já que } \\
\text { deve haver um consenso sobre o conhecimento ali representado. Quando uma mudança é proposta, os usuários são notificados e podem } \\
\text { aceitá-la ou não (Euzenat, 1996). }\end{array}$ \\
\hline (KA) & $\begin{array}{l}\text { Modela formas de aquisiçăo do conhecimento usando ontologias desenvolvidas em conjunto por pessoas em diferentes locais, mas que } \\
\text { utilizam o mesmo padrão; a comunicação e coordenação são feitas via agentes inteligentes (Kietz, Maedche \& Volz, 2000). }\end{array}$ \\
\hline
\end{tabular}

TABELA 14

Metodologias para aprendizado sobre a estrutura de ontologias

\begin{tabular}{|l|l|}
\hline Metodologia & \multicolumn{1}{c|}{ Breve descrição } \\
\hline Maedche & $\begin{array}{l}\text { Uma ontologia genérica é convertida em modelo; especificam-se textos e obtêm-se conceitos do domínio a partir das fontes } \\
\text { disponíveis; removem-se conceitos genéricos, de forma que apenas os conceitos específicos do domínio permaneçam; a estrutura } \\
\text { conceitual da ontologia está estabelecida e obtêm-se as relaçôes. Novas relaçốes conceituais são induzidas por métodos de } \\
\text { aprendizado (Kietz, Maedche \& Volz, (2000). }\end{array}$ \\
\hline
\end{tabular}

TABELA 15

Metodologias para integração de ontologias

\begin{tabular}{|l|l|}
\hline \multicolumn{1}{|c|}{ Metodologia } & \multicolumn{1}{c|}{ Breve descriçäo } \\
\hline FCA-Merge & $\begin{array}{l}\text { Utiliza de processamento de linguagem natural e análise formal de conceitos sobre as ontologias que se deseja integrar, } \\
\text { resultando em um conjunto de conceitos; o resultado é analisado por especialistas e transformado em uma ontologia composta } \\
\text { (Stumme \& Maedche, 2001). }\end{array}$ \\
\hline PROMPT & $\begin{array}{l}\text { Trata-se de um algoritmo aplicável a várias plataformas que integra ontologias e é baseado em um modelo de conhecimento } \\
\text { genérico; executa algumas tarefas automaticamente (existe participaçấo humana) e determina inconsistências na ontologia, } \\
\text { sugerindo como removê-las (Noy \& Musen, 2000). }\end{array}$ \\
\hline
\end{tabular}


TABELA 16

Ferramentas para construção de ontologias

\begin{tabular}{|c|c|}
\hline Ferramentas & Breve descrição \\
\hline $\begin{array}{l}\text { CODE4 (Conceptually Oriented } \\
\text { Description Environment) }\end{array}$ & $\begin{array}{l}\text { Ferramenta de propósito geral que possui diferentes modos de herança e inferência, uma interface gráfica de fácil } \\
\text { uso, um modo de hipertexto para navegação e utilitários para leitura de documentos e gerenciamento léxico (Skuce, } \\
\text { 1995). }\end{array}$ \\
\hline VOID & $\begin{array}{l}\text { Ambiente para navegação, edição e gerenciamento de ontologias. Por meio de simulações, possibilita o estudo de } \\
\text { questões teóricas, como organização de bibliotecas de ontologias e tradução entre diferentes formalismos (Schreiber, } \\
\text { Terpstra \& Sisyphus, 1995). }\end{array}$ \\
\hline $\begin{array}{l}\text { IKARUS (Intelligent Knowledge } \\
\text { Acquisition and Retrieval } \\
\text { Universal System) }\end{array}$ & $\begin{array}{l}\text { Explora as capacidades cooperativas do ambiente Web. Utiliza uma representação hierárquica gráfica que permite } \\
\text { herança múltipla. As declarações que contêm a informação são representadas como predicados com sintaxe e } \\
\text { semântica definidos ou como fragmentos sem estrutura (Skuce, 1996). }\end{array}$ \\
\hline Ontolingua & $\begin{array}{l}\text { Conjunto de serviços que possibilitam a construção de ontologias compartilhadas entre grupos. Permite acesso a uma } \\
\text { biblioteca de ontologias, tradutores para linguagens e um editor para criar e navegar pela ontologia. Editores remotos } \\
\text { podem editar ontologias usando protolocos. (Farquhar, Fikes \& Rice, 1996) }\end{array}$ \\
\hline Ontosaurus & $\begin{array}{l}\text { Consiste de um servidor de ontologias que usa o LOOM para representação do conhecimento e um servidor de } \\
\text { navegação por ontologias que cria páginas HTML dinamicamente e apresenta a hierarquia da ontologia (Swartout et } \\
\text { alii, 1996). }\end{array}$ \\
\hline $\begin{array}{l}\text { GKB-Editor (Generic Knowledge } \\
\text { Base Editor) }\end{array}$ & $\begin{array}{l}\text { Ferramenta para navegação e edição de ontologias por meio de sistemas de representação baseados em frames* } \\
\text { Oferece interface gráfica, em que os usuários podem editar diretamente a base de conhecimento e selecionar a parte que } \\
\text { é de seu interesse (Paley \& Karp, 1997). }\end{array}$ \\
\hline JOE (Java Ontology Editor) & $\begin{array}{l}\text { Ferramenta para construção e visualização de ontologias. Proporciona gerenciamento do conhecimento em } \\
\text { ambientes abertos, heterogêneos e com diversos usuários. As ontologias são visualizadas como um diagrama } \\
\text { entidade-relacionamento, como o gerenciador de arquivos do } M S \text { Windows ou como uma estrutura em árvore } \\
\text { (Mahalingam \& Huhns, 1997). }\end{array}$ \\
\hline $\begin{array}{l}\text { APECKS (Adaptive Presentation } \\
\text { Environment for Collaborative } \\
\text { Knowledge Structuring) }\end{array}$ & $\begin{array}{l}\text { É um servidor de ontologias que permite trabalho cooperativo mediante a criação de ontologias pessoais pelos } \\
\text { usuários. Estas ontologias podem ser comparadas com outras, e é possível a discussão sobre as diferenças e } \\
\text { similaridades entre elas (Tennison \& Shadbolt, 1998). }\end{array}$ \\
\hline OilEd & $\begin{array}{l}\text { É um editor de ontologias de código aberto que permite construir ontologias utilizando a linguagem OIL. Não é um } \\
\text { ambiente completo para desenvolvimento de ontologias. Verificação da consistência e classificação automática da } \\
\text { ontologia podem ser executadas pela ferramenta FaCT (Horrocks, Sattler \& Tobies, 1999). }\end{array}$ \\
\hline OntoEdit & $\begin{array}{l}\text { É um ambiente gráfico para edição de ontologias que permite inspeção, navegação, codificação e alteração de } \\
\text { ontologias. O modelo conceitual é armazenado usando um modelo de ontologia que pode ser mapeado em diferentes } \\
\text { linguagens de representação. As ontologias são armazenadas em bancos relacionais e podem ser implementadas em } \\
\text { XML, FLogic, RDF(S) e DAML+OIL (Maedche et alii, 2000). }\end{array}$ \\
\hline $\begin{array}{l}\text { OCM (Ontological Constraints } \\
\text { Manager })\end{array}$ & $\begin{array}{l}\text { É uma ferramenta para verificar a consistência de ontologias em relação a axiomas ontológicos. É composto por duas } \\
\text { ferramentas de edição que possibilitam verificar a ocorrência de conflitos. (Kalfoglou et alii, 2001). }\end{array}$ \\
\hline Protegé 2000 & $\begin{array}{l}\text { É um ambiente interativo para projeto de ontologias, de código aberto, que oferece uma interface gráfica para edição } \\
\text { de ontologias e uma arquitetura para a criação de ferramentas baseadas em conhecimento. A arquitetura é modulada } \\
\text { e permite a inserção de novos recursos (Noy, Fergerson \& Musen, 2000). }\end{array}$ \\
\hline WebODE & $\begin{array}{l}\text { Ambiente para engenharia ontológica que dá suporte à maioria das atividades de desenvolvimento de ontologias. } \\
\text { A integração com outros sistemas é possível , importando e exportando ontologias de linguagens de marcação } \\
\text { (Arpírez et alii., 2001). }\end{array}$ \\
\hline WebOnto & $\begin{array}{l}\text { Ferramenta que possibilita a navegação, criação e edição de ontologias, representadas na linguagem de modelagem } \\
\text { OCML. Permite o gerenciamento de ontologias por interface gráfica, inspeção de elementos, verificação da } \\
\text { consistência da herança e trabalho cooperativo. Possui uma biblioteca com mais de cem ontologias (Domingue, } \\
\text { 2001). }\end{array}$ \\
\hline Ontomarkup Annotation Tool & $\begin{array}{l}\text { Ferramenta baseada em ontologias que incorporarem informações semânticas em documentos mediante anotações. } \\
\text { Contém um componente de marcação que permite a navegação e a marcação de partes relevantes, um componente } \\
\text { que aprende regras a partir de exemplos e um componente de extração da informação (Vargas-Vera et alii, 2001). }\end{array}$ \\
\hline Onto Annotate & $\begin{array}{l}\text { É uma ferramenta de anotação semi-automática que permite a coleta de informações de documentos e páginas da } \\
\text { Web, criando novos documentos com metadados. Permite a anotação em documentos de HTML estático, Ms-Word } \\
\text { e MS-Excel (Handschuh, Staab \& Madche, 2001). }\end{array}$ \\
\hline $\begin{array}{l}\text { Asium (Acquisition of SemantIc } \\
\text { knowledge Using Machine } \\
\text { learning method) }\end{array}$ & $\begin{array}{l}\text { Auxilia um especialista na aquisição de conhecimento e semântica de textos. Possui uma interface amigável que } \\
\text { auxilia na exploração dos textos e no aprendizado da semântica que não está nos textos, como, por exemplo, de uma } \\
\text { ontologia, que representa os conceitos estudados no domínio (Faure \& N'edellec, 1998). }\end{array}$ \\
\hline Text-to-onto & $\begin{array}{l}\text { Proporciona um ambiente para o aprendizado e construção de ontologias a partir de textos. Os textos podem ser em } \\
\text { linguagem natural ou formatados em HTML. O sistema é composto por um módulo de gerenciamento de textos e } \\
\text { um extrator de informações. Os resultados são armazenados em XML (Maedche \& Volz, 2001). }\end{array}$ \\
\hline
\end{tabular}

* Frames são estruturas de dados que contêm variáveis pertencentes a um escopo. 
TABELA 17

\section{Linguagens para construção de ontologias}

\begin{tabular}{|c|c|}
\hline Linguagens & Breve descricăo \\
\hline CycL & $\begin{array}{l}\text { Linguagem formal que expressa conhecimento por meio de um vocabulário de termos (constantes semânticas, variáveis, } \\
\text { números, seqüências de caracteres etc.) os quais são combinados em expressōes, sentenças e finalmente bases de } \\
\text { conhecimento (Lenat \& Guha, 1990). }\end{array}$ \\
\hline Flogic(Frame Logic) & $\begin{array}{l}\text { Integra frames e lógica de primeira ordem. Trata de uma forma declarativa os aspectos estruturais das linguagens baseadas } \\
\text { em frames e orientadas a objeto (identificação de objetos, herança, tipos polimórficos, métodos de consulta, } \\
\text { encapsulamento etc). Permite a representaçāo de conceitos, taxonomias, relaçōes binárias, funçōes, instâncias, axiomas e } \\
\text { regras (Kifer, Lausen \& Wu, 1990). }\end{array}$ \\
\hline LOOM & $\begin{array}{l}\text { Descendente da família KL-ONE (Knowledge Language One), é baseada em lógica descritiva e regras de produção. } \\
\text { Permite a representação de conceitos, taxonomias, relaçốes n-árias, funçôes, axiomas e regras de produção (Brill, 1993). }\end{array}$ \\
\hline CARIN & $\begin{array}{l}\text { Trata-se de uma combinaçäo da Datalog (linguagem baseada em regras) e lógica descritiva ALN. Uma ontologia CARIN } \\
\text { é construída por dois componentes terminológicos: um conjunto de conceitos com declaraçốes de inclusăo e um conjunto } \\
\text { de regras que usam os conceitos (Levy \& Rousset, 1996). }\end{array}$ \\
\hline GRAIL & $\begin{array}{l}\text { É uma linguagem que especifica uma ontologia do domínio médico (Galen). É uma linguagem baseada em lógica } \\
\text { descritiva, terminologicamente limitada, que permite a construção de hierarquias de primitivas e axiomas de inclusão de } \\
\text { conceitos (Rector et alii, 1997). }\end{array}$ \\
\hline Ontolingua & $\begin{array}{l}\text { Combina paradigmas das linguagens baseadas em frames e logica de primeira ordem. Permite a representaçăo de } \\
\text { conceitos, taxonomias de conceitos, relaçסes n-árias, funç̋es, axiomas, instâncias e procedimentos. Sua alta } \\
\text { expressividade causa problemas na construçăo de mecanismos de inferência (Chaudhri et alii, 1998). }\end{array}$ \\
\hline$O C M L$ & $\begin{array}{l}\text { Permite a especificação de funções, relações e classes, instâncias e regras. Utilizada em aplicações de gerenciamento do } \\
\text { conhecimento, desenvolvimento de ontologias, comércio eletrônico e sistemas baseados em conhecimento. Aplicada em } \\
\text { medicina, ciências sociais, memória corporativa, engenharia, portais da Web etc. (Domingue, Motta \& Corcho, 1999; } \\
\text { Chaudhri, Karp \& Thomere, 1999). }\end{array}$ \\
\hline $\begin{array}{l}\text { OML (Onto } \\
\text { Language) }\end{array}$ & $\begin{array}{l}\text { baseada em lógica descritiva e grafos conceituais que perrmite a representação de conceitos organizados em } \\
\text {, relaçöes e axiomas (Kent, 1999). }\end{array}$ \\
\hline $\begin{array}{l}\text { RDF (Resource Description } \\
\text { Framework)/RDFS(RDF } \\
\text { Schema) }\end{array}$ & $\begin{array}{l}\text { Desenvolvidos pelo W3 Consortium, têm por objetivo a representaçăo de conhecimento por meio da idéia de redes } \\
\text { scmânticas. São linguagens que permitem a representação de conccitos, taxonomias de conccitos c relaçốes binárias } \\
\text { (Lassila \& Swick, 1999). }\end{array}$ \\
\hline $\begin{array}{l}\text { NKRL (Narrative Knowledge } \\
\text { Representation Language) }\end{array}$ & $\begin{array}{l}\text { taçăo baseada em frames especialmente desenvolvida para descrever modelos semânticos de } \\
\text { Bertino, Barbara \& Zarri, 1999). }\end{array}$ \\
\hline $\begin{array}{l}\text { SHOE (Simple HTML } \\
\text { Ontology Extensions) }\end{array}$ & $\begin{array}{l}\text { Utiliza extensões ao HTML, adicionando marcaç̋̄es para inserir metedados em páginas Web. As marcaç̄es podem ser } \\
\text { utilizadas para a construçăo de ontologias e para anotaçōes em documentos da } W e b \text {. (Heflin \& Hendler, 2000). }\end{array}$ \\
\hline$X O L$ & $\begin{array}{l}\text { É uma linguagem que pode especificar conceitos, taxonomias e relaçṍes binárias. Não possui mecanismos de inferência e } \\
\text { foi projetada para a intercâmbio de ontologias no domínio da biomédica (Karp, 1997). }\end{array}$ \\
\hline $\begin{array}{l}\text { OIL (Ontology Interchange } \\
\text { Language) }\end{array}$ & $\begin{array}{l}\text { Precursor do DAML+OIL e base para uma linguagem para a Web Semântica. Combina primitivas de modelagem das } \\
\text { linguagens baseadas em frames com a semântica formal e serviços de inferência da lógica descritiva. Pode verificar } \\
\text { classificação e taxonomias de conceitos (Fensel et alii, 2001). }\end{array}$ \\
\hline $\begin{array}{l}\text { DAML (DARPAAgent } \\
\text { Markup Language) }+ \text { OIL }\end{array}$ & $\begin{array}{l}\text { DAML+OIL é uma linguagem de marcação semântica para a } W e b \text { que apresenta extensões a linguagens como o DAML, } \\
\text { RDF e RDFS, por meio de primitivas de modelagem baseadas em linguagens lógicas. (Horrocks et alii, 2001). }\end{array}$ \\
\hline $\begin{array}{l}\text { FOML (formal ontology } \\
\text { markap language) }\end{array}$ & $\begin{array}{l}\text { Trata-se de uma linguagem de marcação, baseada em XML, que conecta documentos da Web com ontologias formais. } \\
\text { O objetivo é a aquisição automática de conhecimento de domínios específicos (Ogata, 2001). }\end{array}$ \\
\hline
\end{tabular}

TABELA 18

\section{Métodos para avaliação de ontologias}

\begin{tabular}{|l|l|}
\hline \multicolumn{1}{|c|}{ Metodologia } & \multicolumn{1}{c|}{ Breve descriçäo } \\
\hline $\begin{array}{l}\text { WELTY e } \\
\text { GUARINO }\end{array}$ & $\begin{array}{l}\text { É uma metodologia para avaliaçá̃o de taxonomias que utiliza princípios filosóficos. O usuário pode fazer anotaçốes nas } \\
\text { propriedades da taxonomia e depois avaliá-la (Welty \& Guarino, 2000). }\end{array}$ \\
\hline GÓMEZ-PÉREZ & $\begin{array}{l}\text { Avalia erros na construção da ontologia provenientes da estruturação do domínio em taxonomias e bases de conhecimento. Leva } \\
\text { em conta o trabalho realizado previamente na avaliação das ontologias e os critérios utilizados para tal (Gómez-Pérez, 2001). }\end{array}$ \\
\hline
\end{tabular}

\section{ONCLUSÕES}

Este artigo proporcionou uma visão geral sobre o estadoda-arte no estudo de ontologias. A presentaram-se definições, tipos, aplicações, metodologias, ferramentas e linguagens para a construção de ontologias. Pesquisouse 0 uso de ontologias, destacando projetos, repositórios e ontologias conhecidas.
A pesar da preocupação em cobrir os itens mais representativos descritos recentemente na literatura, 0 estudo não é exaustivo e tem se verificado o surgimento de novas iniciativas. Conclui-se que a existência de inúmeras e variadas abordagens justifica um trabalho de sistematização como 0 apresentado.

0 grande volume de pesquisa sobre 0 assunto sugere a importância e a utilidade das ontologias na tarefa de organizar informações em um domínio do 
conhecimento. 0 ambiente informacional atual é mais abrangente do que há alguns anos, principalmente em função do advento da Internet e da popularização dos computadores. Junto a outras ferramentas tradicionais utilizadas pela biblioteconomia (como, por exemplo, os tesauri), as ontologias podem proporcionar melhorias na recuperação da informação ao organizar o conteúdo de fontes de dados que compõem um domínio. Além disso, as ontologias permitem formas de representação baseadas em lógica, o que possibilita o uso de mecanismos de inferência para criar novo conhecimento a partir do existente. D essa forma, representam uma evolução em relação a técnicas tradicionais.

Espera-se que este trabalho sirva de base para estudos que aprofundem algumas das abordagens aqui pesquisadas. As linguagens, ferramentas e metodologias para a construção de ontologias devem ser discutidas e analisadas, para se determinar sua melhor utilização, contribuindo para o avanço da área de representação do conhecimento. Tais tarefas estão além do escopo deste artigo, e pretende-se abordá-las em trabalhos futuros.

\section{REFERÊN C IAS}

AGUADO, G. et al. Ontogeneration: reusing domain and linguistic ontologies for Spanish text generation? In: EU RO PEAN CONFERENCE ON ARTIFICIAL INTELLIGENCE. 13. 1998. Brighton, England, 1998. p. 23-28.

ALBERTAZZI, Liliana. Formal and material ontology. In: POLI, Roberto; SIM ONS, Peter (Ed.). Formal ontology. Dordrecht : Kluwer, 1996. p. $199-232$

ALEXAKI, S. et al. M anaging RDF metadata for community webs. In: INTERNATIONAL WORKSHOP ON THE WORLD WIDE WEB AND CONCEPTUAL MODELING. 2. 2000. [S. I. : s. n.], 2000. p. 140-151. Disponível em: <http://139.91.183.30:9090/RDF/ publications/wcm2000.PDF >. A cesso em: 11 out. 2002.

ARPÍREZ, J. C. et al. Web ODE: a scalable workbench for ontological engineering. In: INTERNATIONAL CONFERENCE ON KN OWLEDGE CAPT URE. PROCEEDINGS. Victoria, British Columbia, Canada, 2001.

BATEMAN, J. A. U sing text structure and text planning to guide text summarization. Disponível em: <http:// www.ik.fhhannover.de/ ik/ projekte/ D agstuhl/ A bstract/A bstracts/ Bateman/ Bateman.html>. Acesso em: 25 maio 2002.

BATEMAN, J.; MAGNINI, B.; FABRIS, G. The generalized upper model knowledge base: organization and use. In: CONFERENCE ON KN OWLEDGE REPRESEN TATION AND SHARING. 1995. Proceedings. Twente, the $\mathrm{N}$ etherlands, 1995.

BEALE, S.; NIRENBURG, S.; MAHESH, K. Semantic analysis in the mikrokosmos machine translation project. In: PROCEEDINGS OF THE SECOND SYMPOSIUM ON NATURAL LANGUAGE PRO CESSING. 1995. Disponível em: <http:// crl.nmsu.edu/ users/ sb/ papers/thai/thai.html>. A cesso em: 6 jul. 2002.

Ci. Inf., Brasília, v. 32, n. 3, p. 7-20, set./dez. 2003
BERNARAS, A.; LARESGOITI, I.; CORERA, J. Building and reusing ontologies for electrical network applications. In: PROCEEDINGS OF THE EUROPEAN CONFERENCE ON ARTIFICIAL IN TELLIGEN CE, 1996. [S. I. : s. n.], 1996. p. 298-302.

BERTIN O, E.; BARBAR A, C.; ZARRI, G. P. A conceptual annotation approach to indexing in a web-based information system. In: INTERNATIONAL WORKSHOP ON ADVANCE ISSUES OF ECOMMERCE AND WEB-BASED INFORMATION SYSTEMS. 1999. Santa Clara, California : [s. n.], 1999.

BLACKBURN, S. Consultoria da edição brasileira. In: MARCONDES, D. Dicionário oxford de filosofia. Tradução D. Murcho et al. Rio de Janeiro : Jorge Zahar, 1997.

$B O N D, F$. et al. Design and construction of a machine - tractable Japanese-M alay lexicon. In: ANNUAL MEETING OF THE ASSOCIATION FOR NATURAL LANGUAGE PROCESSING. 7. 2001. [S. I. : s. n.], 2001.

BORGO, S. et al. U sing a large linguistic ontology for Internet-based retrieval of object-oriented components. In: PROCEEDINGS OF THE INTERNATIONAL CONFERENCE ON SOFTWARE ENGINEERING AND KNOWLEDGE ENGINEERING. 9. 1997. Madrid. Madrid : [s. n.], 1997.

BORST, W. N. Construction of engineering ontologies. 1997. Tese (Doutorado). Disponível em: <http:// www.ub.utwente.nl/ webdocs/ inf/ 1/t0000004.pdf>. A cesso em: 21 abr. 2002.

BRILL, D. Loom reference manual. In: BACON, J. HAYTON; R. M O ODY, K. M iddleware for digital libraries. [S. I. : s. n.], 1998. Disponível em: <http:// www.dlib.org/dlib/october98/bacon/ 10bacon.html>. Acesso em: 15 nov. 2001.

BUITELAAR, Paul. Semantic lexicons: between ontology and terminology. In: PROCEEDINGS OF ONTOLEX: ONTOLOGIES AND LEXICAL KNOWLEDGE BASES. 2000. OntoText Lab. : Sofia, Bulgaria, 2001.

CAPLINKAS, A. ; EDER, J. (Ed.). Advances in databases and information systems. Technika, v. 2. p.29-41, 2001.

CORAZZO N, R. W hat is ontology? [S. I. : s. n.], 2002. Disponível em: $<h t t p: / /$ www.formalontology.it/ section_4.htm>. A cesso em: 20 jul. 2002.

CHANDR ASEKAR AN, B.; JOHNSON, T. R.; BENJAM INS, V. R. Ontologies: what are they? Why do we need them? IEEE Intelligent Systems, v. 14, n. 1, p. 20-26, 1999.

CH AU DHRI, V. K. et al. O pen knowledge base connectivity 2.0. [S. I. : s. n.], 1998. Disponível em: <http:// www-ksl-svc.stanford.edu:5915/ doc/ release/ okbc/ okbc-spec/ okbc-2-0-3. pdf>. A cesso em: 09 jun. 2002.

DOM IN GU E, J. Tadzebao and webonto: discussing, browsing and editing ontologies on the web. In: PROCEEDINGS OF THE 11th BANFF KN OW LED GE ACQUISITION W ORKSH OP. 1998. Banff, Alberta, Canada, [s.n.], 1998.

; M OTTA, E. A Knowledge-based news server supporting ontology-driven story enrichment and knowledge retrieval. In: KNOWLEDGE ACQUISITION, MODELING AND MANAGEMENT. 1999. Dagstuhl Castle, Germany : [s. n.], 1999.

; $\mathrm{CORCHO} 0$. Knowledge modeling in web onto and OCML: a user guide. [ S. I. : s. n.], 1999. Disponível em: <http:// kmi.open.ac.uk/projects/webonto/ user_guide. 2.4.pdf>. A cesso em: 09 jun 2002. 


\section{Mauricio B. Almeida / M arcello P. Bax}

et al. Supporting ontology driven document enrichment within communities of practice. In: PROCEEDINGS OF THE INTERNATIONAL CONFEREN CE ON KN OWLEDGE CAPTURE. 2001. Victoria, British Columbia, Canada. [S. n.], 2001.

EU ZENAT, J. Corporative memory through cooperative creation of knowledge bases and hyper-documents. In: PROCEEDINGS ON KNOWLEDGE ACQUISITION FOR KNOWLEDGE-BASED SYSTEM S WORKSHOP. 10. 1996. Disponível em: <http:// ksi.cpsc.ucal gary.ca/ KA W / KA W 96/ euzen at/ euzen at96b.html >. A cesso em: 28 set. 2001.

FARQ U HAR, A.; FIKES, R.; RICE, J. The ontolingua server: U SA: a tool for collaborative ontology construction. Duluth : A cademic Press, 1997. p. 707-727.

FAU RE; N 'EDELLEC D. ASIU M : learning subcategorization frames and restrictions of selection. In: CONFEREN CE ON M ACH INE LEARN IN G. 10. 1998. Chemnitz, Germany : [s. n.], 1998.

FEN SEL, D. et al. OIL: an ontology infraestructure for the semantic web. [S. I.] : IEEE Intelligent Systems, 2001.

FERNÁNDEZ, M. O verview of methodologies for building ontologies. In: WORKSHOP ON ONTOLOGIES AND PROBLEM -SOLVING METHODS: lessons learned and future trends. 1999. [S. I. : s. n.], 1999. p. 4-1, 4-13.

FERN ÁN DEZ-LÓ PEZ, M . et al. Building a chemical ontology using methotology and the ontology design environment. [S. I.] : IEEE Intelligent Systems \& their A pplications, p. 37-46, Jan./ Feb. 1999.

FOX, M.S. An organizational view of distributed systems. IEEE Transactions on Systems, M an, and Cybernetics, v. 11, n, 1, p. 70-80, 1981.

30. GANDON, F. Engineering an ontology for a multi-agents corporate memory system. In: PROCEEDINS OF THE INTERNATIONAL SYMPOSIUM ON THE MANAGEMENT OF INDUSTRIAL AND CORPORATE KNOWLEDGE. 2001. [S. I. : s. n.], 2001. p. 209-228. Disponível em: <http:// citeseer.nj.nec. com/ gandon01engineering.html>. A cesso em: 22 maio 2002.

GENESERETH, M ichael R.; NILSSO N, L. Logical foundation of AI. San Francisco, Los Altos, Califórnia : M organ Kaufman, 1987.

GERM ANN , U . M aking semantic interpretation parser-independent. In: PROCEEDINGS OF THE AMTA CONFERENCE, 4. 1998. Disponível em: <http:// www.isi.edu/ natural-language/ people/ germann/ >. A cesso em: 11 maio 2002.

GÓM EZ-PÉREZ, A. Evaluation of taxonomic knowledge in ontologies and knowledge bases. In: TWELFTH WORKSHOP ON KNOWLEDGE ACQUISITION, MODELING AND MANAGEMENT, 12. 1999, Alberta, C anadá. [S. I : s. n.], 1999.

; BENJAMINS, V. R. O verview of knowledge sharing and reuse components: ontologies and problem-solving methods. In: PROCEEDINGS OF THE WORKSHOP ON ONTOLOGIES AND PROBLEM-SOLVING METHODS, 1999, Stockholm, Sweden. [S. I. : S. n.], 1999.

GRU BER, T. (1996). W hat is an ontology? [S. I. : s. n.], 1996. Disponível em: <http:// www-ksl.stanford.edu/ kst/ what-is-an-ontology.html>. A cesso em: 14 set. 2002.

GRÜNINGER, M .; FOX, M. S. M ethodology for the design and evaluation of ontologies. In: WORKSHOP ON BASIC ONTOLOGICAL ISSUES IN KNOWLEDGE SHARING, 1995, M ontreal. [S. I. : S. n.], 1995.
37. GUARINO, N. Formal ontology in information systems. In: PROCEEDINGS OF THE INTERNAT. CONFERENCE ON FORMAL ONTOLOGY IN INFORMATION SYSTEMS, 2001, Buffalo. N ova York : Barry Smith U niversity at Buffalo, 2001.

GUARINO, N. Understanding, building and using ontologies. In: PROCEEDINGS OF KNOWLEDGE ACQUISITION FOR KN OW LEDGE-BASED SYSTEMS WORKSHOP. 10. 1996. Disponível em: <http:/ / ksi.cpsc.ucalgary.ca/ KAW / KAW 96/ guarino/ guarino.html\# Heading4>. A cesso em: 22 set. 2001.

; GIARETTA, P. O ntologies and KBs, towards a terminological clarification. In: M ARS, N. (Ed.). Towards a very large knowledge bases: knowledge building and knowledge sharing. [S. I.] : IO S Press, 1995. p. 25-32.

; WELTY, C. A. Formal ontology of properties.In:DIENG,R.;CORBY,O. (Ed.).INTERNATIONAL CONFERENCE ON KNOWLEDGE ENGEINEERING AND KN OW LED GE M AN AGEM ENT: methods, models and tools. 12. 2000. [S. I.] : Springer Verlag, 2000. p. 97-112.

HAAV, H. M.; LUBI, T. L. A survey of concept-based information retrieval tools on the web. In: PROCEEDINGS OF EAST-EU ROPEAN CONFEREN CE ADBIS. 5. 2001.

HANDSCHU H, S.; STAAB, S.; M ADCHE, A. CREAM -creating relational metadata with a component-based,ontology-driven annotation framework. [S. I. : s. n.], 2001.

HASM AN, A. et al. ID2.1: A nalysis of guideline ontologies. [S. I. : S. n.], 1999. Disponível em: <http:// new.euromise.org/ mgt/ repdev/ id21.html>. A cesso em: 29 ago. 2002.

HEFLIN, J.; HENDLER, J. Searching the web with SHOE. In: ARTIFICIAL INTELLIGENCE FOR WEB SEARCH. 2000. Menlo Park: AAAI Press, CA, p. 35-40, 2000.

HORROCKS, I.; SATTLER, U .; TOBIES, S. Practical reasoning for expressive description logics. Logic Journal of the IGPL, v. 8, n. 3, p. 239-264, may 2000.

et al. The ontology interchange language (OIL). [S. I. : s. n.], 2001. Disponível em: <http:// public.research.mimesweeper.com/ Standards/ W 3C/RDF-related/ oil.pdf>. A cesso em: 19 out.2002.'

et al. Reference description of the DAM L+O IL ontology markup language. [S. I. : s. n.], 2001. Disponível em: <http:// www.w3.org/ TR/daml+oil-reference>. A cesso em: 04 nov. 2001.

JASPER, R.; USCHOLD, M. A framework for understanding and classifying ontology applications. IJCAI-99, ON TOLOGY WORKSH OP, 1999, Stockholm, [S. I. : s. n.], 1999.

JIN, L. et al. Role explication of simulation in intelligent training systems by training task ontology. In: WORKSHOP ON ARCHITECTURES FOR INTELLIGENT SIMULATION -BASED LEARN IN G EN V IRONMENTS, 1997, Kobe, Japan. [S. I. : s. n.], 1997.

JONES, D.; BEN CH-CAPON, T.; VISSER, P. M ethodologies for ontology development. [S. I. : s. n.], 1998. Disponível em: 〈http:// cweb.inria.fr/ Resources/ONTOLOGIES/methodo-for-onto-dev.pdf>. Acesso em: 17 mar. 2002.

KALFOGLOU, Y. et al. M yPlanet: an ontology-driven web-based personalized news service. In: PROCEEDINGS OF THE IJCAI-01 WORKSHOP ON ONTOLOGIES AND INFORMATION SHARING SEATTLE, 2001. [S. L. S. : S. N.], 2001. p. 44-52. 


\section{U ma visão geral sobre ontologias: pesquisa sobre definições, tipos, aplicações, métodos de avaliação e de construção}

KARP, R.; CHAUDHRI, V.; THOMERE, J. XOL: an XML-based ontology exchange language. [S. I. : S. n.]. 1999. Disponível em: <http://www.ai.sri.com/ pkarp/ xol/ xol.html>. Acesso em: 22 jun. 2002.

KENT, R. E. Conceptual knowledge markup language: the centra core. In: PROCEEDINGS OF THE TWELFTH WORKSHOP ON KNOWLEDGE ACQUISITION, MODELING AND MANAGEMENT, 1999, Banff. [S. I. : S. n.], 1999.

KIETZ, J.; MAEDCHE, A.; VOLZ, R. A method for semi-automatic ontology acquisition from a corporate intranet. In: PROCEEDINGS OF THE EKAW WORKSHOP ON ONTOLOGIES AND TEXTS 2000. [S. I. : s. n.], 2000. v. 51. Disponível em: <http:// www.irit.fr/ ACTIVITES/ EQ_SMI/GRACQ/W SEKAW 2000/PAPERS/ $M$ aedche.pdf>. A cesso em: 29 set. 2001.

KIFER, M.; LAUSEN, G.; WU , J. Logical foundations of objectoriented and frame-based languages. Journal of ACM , v. 42, p. 741843, 1995.

LASSILA, 0.; SWICK, R. Resource D escription Framework (RDF) model and syntax specification. W 3 C recommendation. [ S. I. : s. n.], 1999 Disponível em: <http:// www.w3.org/TR/REC -rdf-syntax/ >. A cesso em: 04 nov. 2001.

LEGER, A et al. O ntology domain modeling support for multilingual services in e-commerce. In: M KBEEM. PRESENTATION SEM IN AR ECAI, 2000 Berlin. [S. I. : s. n.], 2000. Disponível em: বhttp://mkbeem.elibel.tm.fr/ paper/ ecai00-final.pdf>. A cesso em: 22 dez. 2001.

LEN AT, D. B.; GUHA, R.V. Building large knowledge-based systems. M assachussets : Addison-W esley, 1990. 372 p.

LEV Y, A. Y.; ROU SSET, M. C. CARIN : A Representation language integrating rules and description logics. In: PROCEEDINGS OF THE EUROPEAN CONFERENCE ON ARTIFICIAL INTELIGENCE, 1996, Budapest, Hungary. [S. I. : s. n.], 1996.

MAEDCHE, A.; VOLZ, R. The text-to-onto ontology extraction and maintenance environment to appear. In: PRO CEEDIN GS OF THE ICDM WORKSHOP ON INTEGRATIN DATA MINING AND KN OW LED GE MANAGEM ENT, 2001, San Jose, California. [S. I. : s. n.], 2001.

et al. Semantic portal: the SEAL approach to appear: In : FEN SEL, D. et al. (Ed.). C reating the semantic web. Cambridge : M assachussets Institute of Technology, 2001.

MAHALINGAM , K.; HUHNS, M. N. An ontology tool for query formulation in an agent-based context. In: IFCIS INTERNATIONAL CONFERENCE ON COOPERATIVE INFORMATION SYSTEMS, 2., 1997, Kiawah Island, SC. [S. I. : s. n.], 1997. p. 170.

MARTIN, P. H.; EKLUND, P. Large-scale cooperatively-built heterogeneous KBs. In: ICCS'01 INTERNATIONAL CONFEREN CE ON CON CEPTUAL STRU CTURES, 9., 2001. [ S. I. : s. n.], 2001 Disponível em: <http://meganesia.int.gu.edu.au/ phmartin/ W ebKB/doc/ papers/iccs01/ >. A cesso em: 6 set. 2002.

MILLER, George A. WordN et: a lexical database for English Communications of the ACM , v. 38, n. 11, p. 39-41, nov. 1995.

MIZO GU CHI, R.; VAN W ELKEN HU YSEN, J.; IKEDA, M. Task ontology for reuse of problem solving knowledge. In: PROCEEDINGS OF ECAI'94 TOWARDS VERY LARGE KNOWLEDGE BASES, 1994, A msterdam. [S. I.] : IOS Press, 1995, p. 46-59.
M ORENO, A. O.; HERNÁNDEZ, C. P. Reusing the mikrokosmos ontology for concept-based multilingual terminology databases. In: IN TERNATIONAL CONFERENCE ON LANGUAGE RESOURCES \& EVALUATION, 2., 2000, Athens. [S. I. : s. n.], 2000.

NECHES, R. et al. Enabling technology for knowledge sharing. AI M agazine, v. 12, n. 3, Fall 1991.

NOY, F. N.; GUIN NESS, D. L. O ntology development 101: a guide to create your first ontology. Disponível em: <http:// ksl.stanford.edu/ people/ dlm/ papers/ ontology-tutorial-noy-mcguinness. doc>. A cesso em: 04 maio 2001.

NOY, N. F.; MUSEN, M. A. PROMPT: algorithm and tool for automated ontology merging and alignment. In: NATIONAL CONFERENCE ON ARTIFICIAL INTELLIGENCE, 12., 2000. [S. I. : s. n.], 2000. p. 450-455. NATIONAL CONFERENCE ON ARTIFICIAL INTELLIGENCE, 17., Austin, TX, 2000. [S. I. : s. n.], 2000.

OGATA, N. A formal ontology discovery from web documents. Web intelligence: research and development. In: PROCEEDINGS LECTURE NOTES IN COMPUTER SCIENCE, 2198, 2001 AND ASIA-PACIFIC CONFERENCE, 1. M aebashi City, Japan. [S. I.] : Springer, 2001.

OGIN O, T. et al. An experiment on matching EDR concept classification dictionary with wordnet. IJCAI-97 WORKSHOP WP24 ONTOLOGIES AND MULTILINGUAL, 1997. [S. I. : s. n.], 1997.

PALEY, J. L. S.; KARP, P. A Generic knowledge base browser and editor. In: PROCEEDINGS OF THE NATIONAL CONFERENCE ON ARTIFICIAL INTELLIGENCE, 14, 1997 AND INNOVATIVE APPLICATIONS OF ARTIFICIAL INTELLIGENCE CONFERENCE, 9., 1997, Providence, Rhode Island. [S. I.] : MIT, 1997. p. 10451051

PISANELLI, D.; Gangemi, A.; STEVE. G. An ontological analysis of the U M LS methatesaurus. In: PROCEEDINGS OF AMIA , 1998. [S. I. : s. n.], 1998.

RECTOR, A. et al.Terminology server for medical language and medical information systems. M ethods of Information in M edicine, v. 34, p. 147-157. 1995.

RÖSNER, D.; STEDE, M. Techdoc: multilingual generation of online and off line instructional text online and offline instructional text. [S. I. : s. n.], 1994 Disponível em: <http:// acl.Idc.upenn.edu/A / A 94/ A 94-1044.pdf >. Acesso em: 22 jul. 2002.

SCHREIBER, A.; TERPSTR A, P.; SISYPHU S,V.T. A common KADS solution. [S. I. : S. n.], 1995. (Technical report). Disponível em: «http:/ / www.swi.psy.uva.nl/ projects/Kactus/ Papers.html>. A cesso em: 28 jun. 2002.

SHUM, S. B. E, MOTTA; DOMINGUE, J. ScholOnto: An ontologybased digital library server for research documents and discourse. International Journal on Digital Libraries, v. 3, n. 3, p. 237-248, Sept. 2000.

SKUCE, D. IKARUS: intelligent knowledge acquisition and retrieval universal system. [S. I. : s. n.], 1996. Disponível em: <http:// www.csi.uottawa.ca/ -kavanagh/l karus/ I karus4.html>. A cesso em: 04 out. 2002

CODE4: a unified system for managing conceptual knowledge. International Journal of Human-C omputer Studies, n. 42, p. 413-451, 1995. 


\section{Mauricio B. Almeida / M arcello P. Bax}

SOW A, J. F. Building, sharing and merging ontologies. Tutorial. [S. I. : s. n.], 1999. Disponível em: <http:/ / users.bestweb.net/ sowa/ ontology/ontoshar.htm>. A cesso em: 30 maio 2002.

Knowledge representation: logical, philosophical, and computational foundations. Pacific Grove, CA : Brooks Cole Publishing, 1999.

STAAB, S. et al. Knowledge processes and ontologies. Intelligent systems. IEEE, v. 16, n. 1, p. 26-34, Jan./Feb. 2001.

STUMME, G .; MAEDCHE, A. FCA-merge: bottom-up merging of ontologies. In: INTERNATIONAL JOINT CONFERENCE ON ARTIFICIAL INTELLIGENCE, Seattle. Knowledge Representation and Reasoning, v. 1, 2001.

SWARTOUT, B. et al. Toward distributed use of large-scale ontologies. In: PROCEEDINGS OF AAAI97 SPRING SYMPOSIUM SERIES WORKSHOP ON ONTOLOGICAL ENGINEERING, 1997. [S. I.] : AAAI Press, 1997. p. 138-148.

TENNISON , J.; SH ADBOLT, N. R. APECKS: a tool to support living ontologies. In: PROCEEDINGS OF THE 11th BANFF KN OW LEDGE ACQUISITION W ORKSHOP, 11., 1998, Banff, Alberta, Canada. [S. I.: s. n.], 1998, p. 18-23.

USCHOLD, M.; GRUNINGER, M. O ntologies: principles, methods an applications. Knowledge Engineering Review, v. 11, n. 2, 1996.

KING, M. Building ontologies: towards a unified methodology. In: ANNUAL CONFERENCE. OF THE BRITISH COMPUTER SOCIETY SPECIALIST GROUP ON EXPERT SYSTEM S, 16., 1996, Cambridge, UK. [S. I. : s. n.], 1996. et al. The enterprise ontology? Knowledge Engineering Review, v. 13, 1998. Special issue on Putting ontologies to use.

VAN HEIJIST, G.; SCHREIBER, A. T.; WIELINGA, B. J. U sing explicit ontologies in KBS development. International Journal of $\mathrm{H}$ uman-C omputer Studies, v. 46, n. 2-3, p. 183-192, feb./ march 1997.

VARGAS-VERA M aria et al. Knowledge Extraction by using an ontologybased Annotation tool. In: PROCEEDINGS OF THE WORKSHOP KNOWLEDGE MARKUP AND SEMANTIC ANNOTATION, 2001, Victoria Canada, 2001.

VÁZQ U EZ, E.; V A LER A, F.; BELLIDO , L. M odelado de servicios complejos en uma plataforma de litermediación para comercio electrónico. [S. I. : S. n.], 2001. Disponível em: <http:// www. telecom.ece.ntua.gr/smartec/ documentation/Publications/smartec-jitel2001.pdf>. A cesso em: 14 ago. 2002.

VOSSEN, P. (Ed.). EuroW ordN et: A M ultilingual Database with Lexical Semantic N etworks, Kluwer A cademic Publishers, Dordrecht, 1998.

WACHE, $\mathrm{H}$. et al. O ntology-based integration of information - a survey of existing approaches. In: IJCAI, WORKSHOP ON ONTOLOGIES AND INFORMATION SHARING, 2001. [S. I. : s. n.] Disponível em: <http:// citeseer.nj.nec.com/ wache01ontologybased.html>. A cesso em: 01 maio 2002.

WELTY, C.; GUARIN O, N. Supporting ontological analysis of taxonomic relationships. Data \& Knowledge Engineering, v. 39, n. 1, o ct. 2001.

ZDRAHAL, Z. et al. Sharing engineering design knowledge in a distributed environment. Behaviour and Information Technology, v. 19, n. 3, p. $189-200,2000$. 